

\title{
First-principles calculations of Raman spectra in Li-doped Si nanocrystals
}

\author{
N. Scott Bobbitt \\ Department of Chemical Engineering, \\ The University of Texas at Austin, Austin, Texas 78712, USA \\ James R. Chelikowsky \\ Center for Computational Materials, \\ Institute for Computational Engineering and Sciences \\ Departments of Physics and Chemical Engineering, \\ The University of Texas at Austin, Austin, Texas 78712, USA
}

(Dated: January 7, 2016)

\begin{abstract}
We examine the vibrational and Raman spectra for Li doped Si nanocrytals using real-space pseudopotentials constructed within density functional theory. We calculate differences in the Raman spectra using the Placzek approximation. The insertion of Li atoms into Si nanocrystals disrupts the Si crystal structure forming a region of Li-Si alloy in which the regular cyrstral structure is significantly disrupted. The Raman spectrum for this alloy exhibits a Li induced peak at $440-480 \mathrm{~cm}^{-1}$. We find an accompanying reduction in the size of the dominant bulk-like $\mathrm{Si}$ peak at $520 \mathrm{~cm}^{-1}$. Both of these results are consistent with experiment. Our analysis of the calculated spectrum confirms the utility of using Raman spectroscopy, coupled with first principle computations, to predict the structural and electronic properties of Li doped Si nanocrystals.
\end{abstract}


Nanocrystalline silicon is an important electronic material in the construction of high capacity lithium ion batteries. [1-3] In particular, silicon has a high specific charge capacity. However, anodes made from bulk Si are mechanically weak and prone to fracture. In contrast, nanostructures can withstand high stress levels making them better suited for use as anodes in Li ion batteries. Unfortunately impurities such as $\mathrm{Li}$ behave differently in nanostructures than in bulk crystals. [4-7] This makes modeling the behavior of Li doped Si as function of size somewhat problematic. Specifically, a fundamental understanding of the behavior of Li in nanocrystalline $\mathrm{Si}$ is crucial for fully realizing the potential of this material as an energy storage device.

One useful technique for characterizing the properties of nanostructured Si materials is Raman spectroscopy. [8-10] Raman is a well established experimental technique for characterizing a wide range of materials. It is highly sensitive to both the atomic and electronic structure of the substrate. Consequently, materials have unique Raman signatures, which can be used in identifying different species.

Popular methods of predicting and interpreting experimental Raman spectra for nanocrystals, such as the Richter, Wang, and Ley (RWL) method or the bond polarizability approximation, [11, 12] rely on empirical parameters from bulk crystals. The transferability of these techniques to nanocrystals is sometimes unreliable, partially due to a failure to account for surface features. [13] Also, in bulk crystals, the phonon modes can be delocalized over a large number of bonds, but in a nanocrystal, confinement effects restrict the mode to a smaller area. Concerns about the validity of these phenomenological methods can be addressed by calculating the Raman spectra from first principles using pseudopotential constructed within density functional theory (DFT).

This first-principles technique has been used to study vibrational and spectral properties in a variety of systems, such as silica [14], hafnium oxide. [15], as well as 2D materials like $\mathrm{MoS}_{2}$ [16] and graphene. [17]. Jensen and Schatz [18] used time-dependent DFT to study resonance Raman scattering in fluroescent dye molecules. Han and Bester [19] have used a first principle technique coupled with the RWL method to examine vibrational properties and Raman shifts in group III-V and II-VI nanocrystals. DFT has also been used to examine size dependence of IR spectra for Si nanocrystals. 20]

Previous work from our group on calculating Raman spectra within DFT has discussed the use of Raman spectroscopy as a tool for characterizing nanocrystal size [21] and the 
position of defects within a nanocrystal [22]. Motivated by this and previous work [23] on diffusion of $\mathrm{Li}$ in Si nanostructures, we examine the effects of Li impurities on the crystal structure of Si nanocrystals by calculating the vibrational and Raman spectra of Li-doped Si nanocrystals.

Vibrational modes are calculated from the dynamical matrix, which is constructed using finite differences by displacing each atom along each Cartesian axis and taking the difference in forces. [24] The eigenvalues and eigenmodes of this matrix represent the vibrational frequencies and modes. The nonresonant Raman spectrum can be calculated from the vibrational modes using the Placzek approximation. The contribution of each mode to the Raman tensor, $\alpha^{n}$, is proportional to the change in the dielectric susceptibility, $\chi_{i j}$ associated with the motions of the specified mode. In this way, the Raman spectrum is dependent on changes in both the atomic and electronic structure. We can express the Raman tensor as

$$
\alpha_{i j}^{n}=\sum_{I k} \frac{\partial \chi_{i j}}{\partial r_{I k}} \frac{\nu_{I k}^{n}}{\sqrt{M_{I}}}
$$

where $\nu^{n}$ is the vibrational mode eigenvector, $M_{I}$ is the atomic mass, and $r_{I k}$ represents the nuclear coordinates. Capital letter indices indicate the atomic species index and lowercase indices represent the Cartesian directions.

The derivative $\frac{\partial \chi_{i j}}{\partial r_{I k}}$ can be represented as a second derivative of atomic force with respect to the applied electric field, which can be evaluated numerically using finite differences in a straightforward fashion. [25, 26] The Raman intensity is calculated as a weighted sum of two key values: the mean polarizability, $\bar{\alpha}$, and the anisotropy, $\bar{\beta}$, both of which are calculated as sums of elements of the Raman tensor. These values are averaged over all spatial orientations, and the Raman intensity is then proportional to $I_{\operatorname{Raman}}=45 \bar{\alpha}^{2}+7 \bar{\beta}^{2}$. [27, 28] The contributions from the passivating $\mathrm{H}$ atoms are projected out of the vibrational density of states (VDOS).

Computations were performed using the PARSEC code [29 32, a real-space package that employs Troullier-Martins pseudopotentials [33] and solves the Kohn-Sham equations selfconsistently on a uniform grid, using a higher order finite difference expression for the kinetic energy operator [34]. Convergence of the self-consistency cycle is accelerated by using damped Chebyshev polynomial filtering subspace iteration. This method requires 
an explicit eigenvalue solution for only the initial self-consistent iteration [34, 35]. The exchange-correlation functional is approximated using the local density approximation. [36]

We use a grid spacing of 0.30 a.u. $(1$ a.u. $=0.529 \AA)$. This is a fine spacing, roughly equal to a plane wave cutoff of $50 \mathrm{Ry}$, which we find to be sufficient to converge the total energy within $\sim 0.01 \mathrm{eV} /$ atom and calculate accurate interatomic forces for silicon nanoparticles. Spherical boundary conditions are used, i.e., the wave functions are equal to zero outside of a domain. The domain is constructed such that a vacuum space of 8.0 a.u. exists from the outermost atoms of the cluster to the domain boundary. Structural relaxations were performed until the maximum force on any atom was less than $0.0005 \mathrm{Ryd} / \mathrm{a} . \mathrm{u}$.

The nanocrystals considered in this study were constructed from bulk crystalline silicon by carving out a cluster of a given radius with a $\mathrm{Si}$ atom centered at the origin. The outer most Si atoms were at most two-fold coordinated. Dangling bonds were passivated with hydrogen. [37] The nanocrystal geometries were relaxed and vibrational modes were calculated from relaxed structures. Li impurities were added at tetrahedral interstitial sites [23] near the central atom and near the surface. Ball-and-stick diagrams of the nanocrystals, with selected atoms near the defect site labeled, are shown in Fig. 1

Previous work by Khoo, et al., [22] demonstrated that substituted impurities such as P in Si have little effect on the vibrational spectrum, but change the Raman spectrum owing to changes in electronic structure. However, interstitial impurities such as Li strain the lattice of the Si crystal, and therefore affect the frequencies of the vibrational modes associated with stretching these distorted bonds. Tables I] and II shows the changes in bond lengths and angles in a $\mathrm{Si}_{35} \mathrm{H}_{36}$ nanocrystal due to adding one interstitial $\mathrm{Li}$ atom near the central Si atom.

The bond lengths of the Si-Si bonds neighboring the Li impurity increase by $1.5-3.2 \%$, which is consistent with experimental observations that the Si crystal volume expands during lithiation. [38, 39] Furthermore, the introduction of the Li impurity changes the bond angles of neighboring Si atoms. In the intrinsic nanocrystal, the Si bond angles are uniformly about 109 degrees in agreement with the bulk crystal structure; however, in the lithiated nanocrystal, the correspoding bond angles vary from 105.9 degrees to 113.8 degrees. This indicates that the Li dopant significantly strains the Si crystal lattice, and forms a locally disrupted region near the impurity. This change in the crystal structure induces a concomitant change in the vibrational and Raman spectra, as seen in Fig. 2 and 3 . The formation 
TABLE I: Bond lengths in a.u. between selected atoms for $\mathrm{Si}_{35} \mathrm{H}_{36}$ and $\mathrm{Si}_{35} \mathrm{H}_{36} \mathrm{Li}$ nanocrystals, and percent changes. All the bond lengths are lengthened by 1.5-3.2 percent, indicating an expansion of the Si crystal due to the addition of Li. Atoms are labeled in Fig. 1.

\begin{tabular}{|c|c|c|c|}
\hline Bond & $\mathbf{S i}_{35} \mathbf{H}_{36}$ & $\mathbf{S i}_{35} \mathbf{H}_{36}: \mathbf{L i}$ & percent change \\
\hline $1-2$ & 4.455 & 4.599 & 3.22 \\
$2-3$ & 4.447 & 4.518 & 1.60 \\
$3-4$ & 4.438 & 4.508 & 1.57 \\
$4-5$ & 4.435 & 4.534 & 2.24 \\
$5-6$ & 4.435 & 4.534 & 2.24 \\
$6-7$ & 4.438 & 4.508 & 1.57 \\
$7-2$ & 4.447 & 4.518 & 1.60
\end{tabular}

TABLE II: Bond angles for selected atoms in $\mathrm{Si}_{35} \mathrm{H}_{36}$ and $\mathrm{Si}_{35} \mathrm{H}_{36} \mathrm{Li}$ nanocrystals. Angles are given in degrees, and atoms are labeled in Fig. 1. The distortion of the bond angles indicates that the Li dopant disrupts the regular Si crystal lattice.

\begin{tabular}{|c|c|c|c|}
\hline Angle & $\mathbf{S i}_{35} \mathbf{H}_{36}$ & $\mathbf{S i}_{35} \mathbf{H}_{36}: \mathbf{L i}$ & percent change \\
\hline 012 & 109.5 & 107.8 & -1.50 \\
123 & 109.0 & 105.9 & -2.80 \\
234 & 109.7 & 113.8 & 3.73 \\
345 & 109.8 & 107.5 & -2.07 \\
456 & 109.8 & 113.1 & 3.04 \\
567 & 109.8 & 107.5 & -2.07 \\
672 & 109.7 & 113.8 & 3.73
\end{tabular}

of a Li-Si alloy has been experimentally observed in heavily lithiated Si thin films. [40]

The VDOS for the $\mathrm{Si}_{35} \mathrm{H}_{36}$ nanocrystal shows two prominent peaks: a broad, low frequency acoustic peak around 150-200 $\mathrm{cm}^{-1}$ and a large high-frequency optical peak at 500 $\mathrm{cm}^{-1}$. This latter peak is characteristic of crystalline silicon, although in this small nanocrystal it is red-shifted from its bulk frequency of $520 \mathrm{~cm}^{-1}$. [21] Fig. 2] shows the introduction of $\mathrm{Li}$, which strains the crystalline structure of $\mathrm{Si}$, results in a reduction of this characteristic peak. A new broader peak appears at a lower frequency, around $440 \mathrm{~cm}^{-1}$. This new peak 

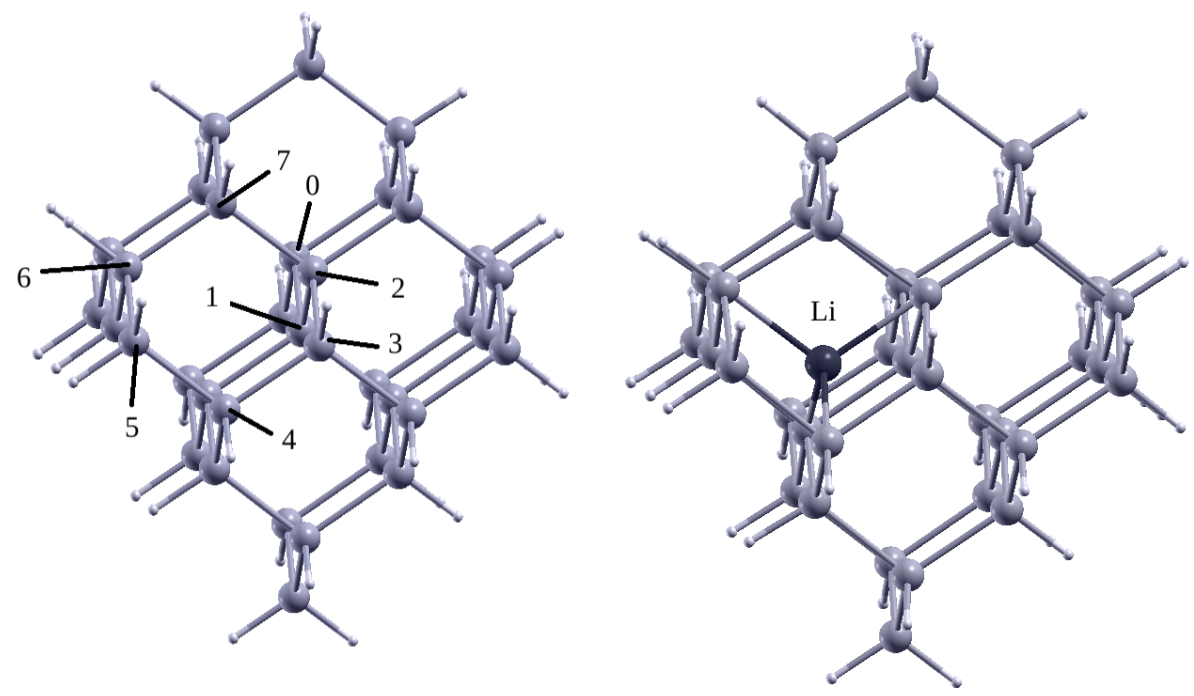

FIG. 1: Ball-and-stick diagrams showing $\mathrm{Si}_{35} \mathrm{H}_{36}$ and $\mathrm{Si}_{35} \mathrm{H}_{36} \mathrm{Li}$ nanocrystals with selected atoms labeled to indicate reported bond lengths and angles.

indicates the irregular Si lattice created by the Li dopant disrupting the Si-Si bonds.

These two key changes in the VDOS are manifested in the Raman spectrum, as shown in Fig. 3 The low frequency acoustic peak is not Raman active, and therefore does not appear in the Raman spectrum. The large peak at $500 \mathrm{~cm}^{-1}$ is significantly reduced by the inclusion of $\mathrm{Li}$, and a new broad peak appears from $420-480 \mathrm{~cm}^{-1}$. This agrees with experimental observations by $\mathrm{Li}$, et al., who report a reduction in the large optical Si peak and the formation of a new broad peak around $480 \mathrm{~cm}^{-1}$. [41], as well as work by Holzapfel, et al., who report similar findings. [42].

Experimental studies have reported a broad Raman peak at $480 \mathrm{~cm}^{-1}$ in amorphous silicon films, [43, 44] which agrees with our calculation of the Raman peak associated with the locally disrupted region surrounding the Li impurity. This suggests that the local disruption of the Si lattice we find near the Li atom behaves similarly to an amorphous Si region within the nanocrystal. The Li atom and surrounding Si atoms form a proto-amorphous alloy, and as more $\mathrm{Li}$ is added to the system we expect that the nanocrystal would more closely resemble amorphous Si.

We find similar results for the $\mathrm{Si}_{87} \mathrm{H}_{76}$ nanocrystal. The addition of one Li atom reduces the large peak at $500 \mathrm{~cm}^{-1}$ in the VDOS, shown in Fig. 4 and the Raman spectrum (Fig. 5). In the larger cluster, the distorted Si framework does not create a distinct new Raman 


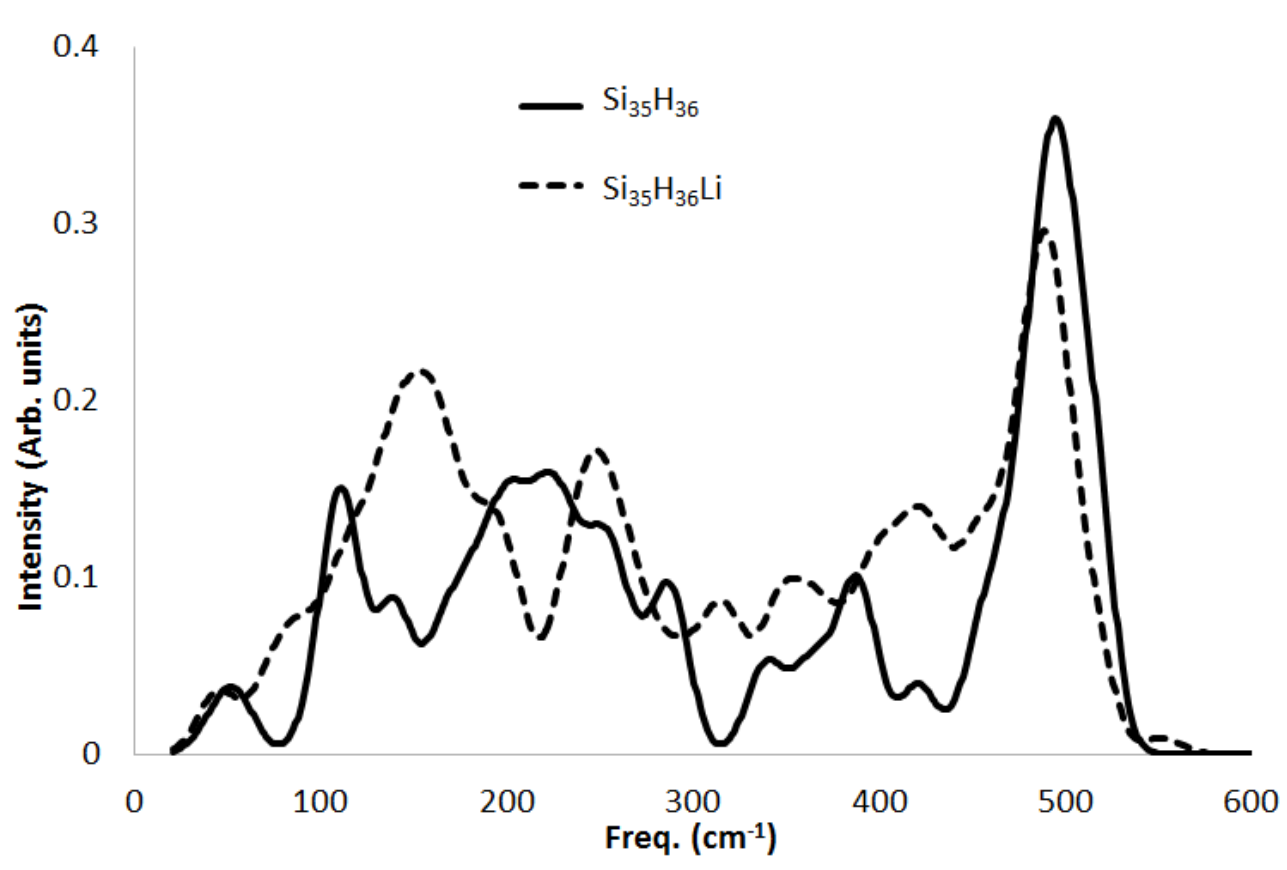

FIG. 2: Vibrational density of states (VDOS) for $\mathrm{Si}_{35} \mathrm{H}_{36}$ and $\mathrm{Si}_{35} \mathrm{H}_{36} \mathrm{Li}$ nanocrystals. $\left(10 \mathrm{~cm}^{-1}\right.$ broadening)

peak, but is instead manifested as a shoulder, or broadening of the Si peak towards lower frequencies, in the range of $450-480 \mathrm{~cm}^{-1}$. We also calculate the VDOS and Raman spectrum for $\mathrm{Si}_{87} \mathrm{H}_{76}$ with four Li dopants, inserted interstitially surrounding the central Si atom. The addition of more Li into the nanocrystal further reduces the size of the crystalline Si peak at $500 \mathrm{~cm}^{-1}$ and the disrupted peak at $465 \mathrm{~cm}^{-1}$ becomes more pronounced because more of the crystal lattice is converted into the locally disrupted region. This dependence of the height of the characteristic peak of crystalline silicon at $500 \mathrm{~cm}^{-1}$ on the number of $\mathrm{Li}$ impurities suggests that Raman spectroscopy could be used to measure the concentration of dopants in silicon nanocrystals by giving an indication of the distortion in the intrinsic crystal lattice.

It is clear from inspection that as more Li atoms are added to the Si crystal the crystalline $\mathrm{Si}$ peak is reduced, and the locally disrupted Si peak grows. We examine this trend by integrating the calculated Raman spectrum over two regions: from $440-480 \mathrm{~cm}^{-1}$, which is associated with the disrupted Si region, and from $480-520 \mathrm{~cm}^{-1}$, which is associated with the crystalline Si region. Fig. 6 shows that for the undoped crystal, the resulting area in the $\mathrm{Si}$ crystal region is four times larger than the disrupted region. As the concentration of $\mathrm{Si}$ 


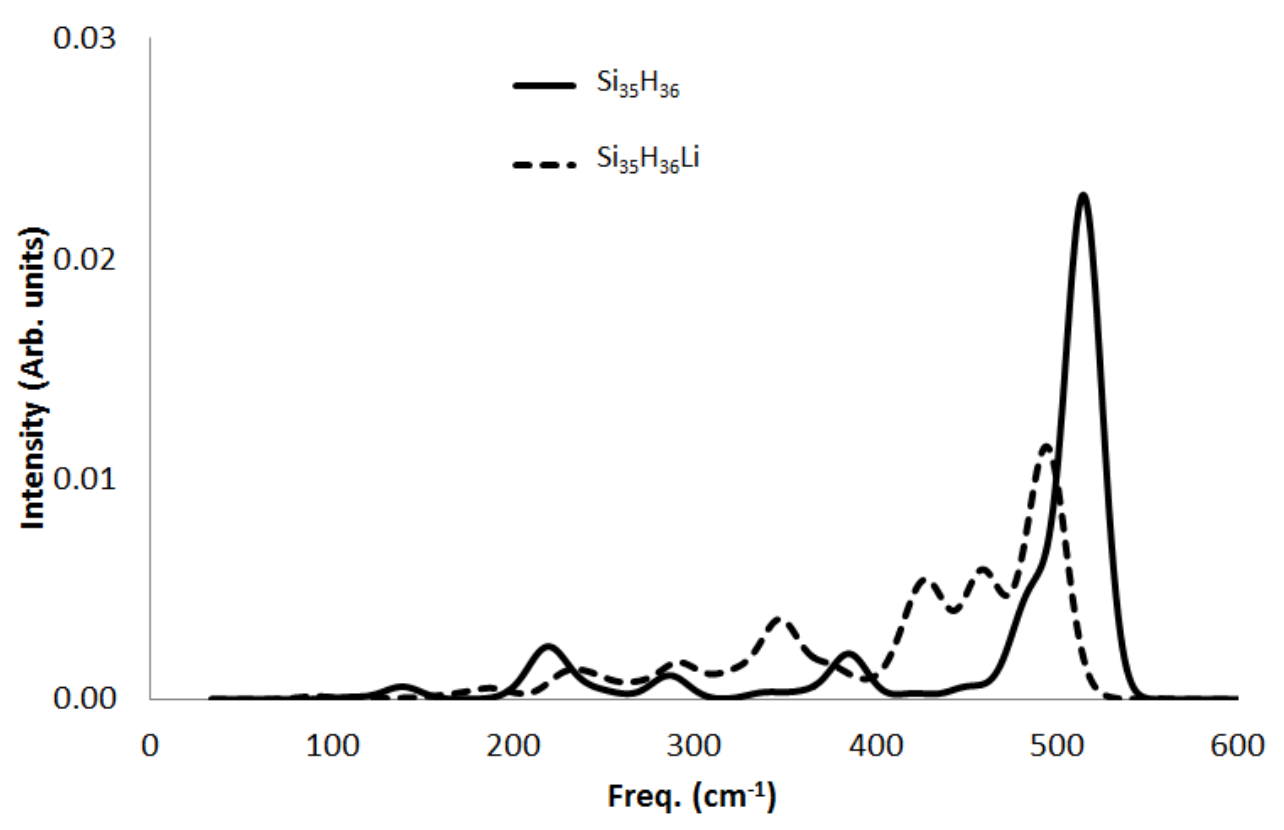

FIG. 3: Calculated Raman cross section for $\mathrm{Si}_{35} \mathrm{H}_{36}$ and $\mathrm{Si}_{35} \mathrm{H}_{36} \mathrm{Li}$ nanocrystals. The broad peak that appears at $420-480 \mathrm{~cm}^{-1}$ indicates the formation of a locally disrupted crystal region near the impurity. (10 $\mathrm{cm}^{-1}$ broadening)

grows, the prominence of the Si crystal is reduced and the disrupted region becomes larger. When the Li concentration is around 4 atomic percent, the disrupted region peak area is nearly twice as large as the Si crystal peak. Extrapolating this trend to a very heavily doped nanocrystal, we expect that the Si crystal peak would disappear entirely, leaving only the disrupted region peak, which has been observed in experiment. 41] Fig. 6 also shows that as the $\mathrm{Li}$ concentration increases, there is a corresponding increase in the number of Si-Si bonds that are distorted by more than 0.5 percent from their intrinsic values. This gives an estimate of what volume fraction of the Si crystal is converted to the locally disrupted state by the lithiation process.

We also find that Li dopants produce different Raman signatures based on the location of the impurity in the nanocrysta, which corroborates previous work by Khoo, et al., [22] Fig. 7 shows that a single Li atom placed on the surface of the $\mathrm{Si}_{87} \mathrm{H}_{76}$ nanocrystal produces a different Raman spectrum from a Li atom placed near the center of the crystal. The peak associated with the locally disrupted region is redshifted in the spectrum with a surface impurity compared to the spectrum of the central impurity. This implies that the distorted 


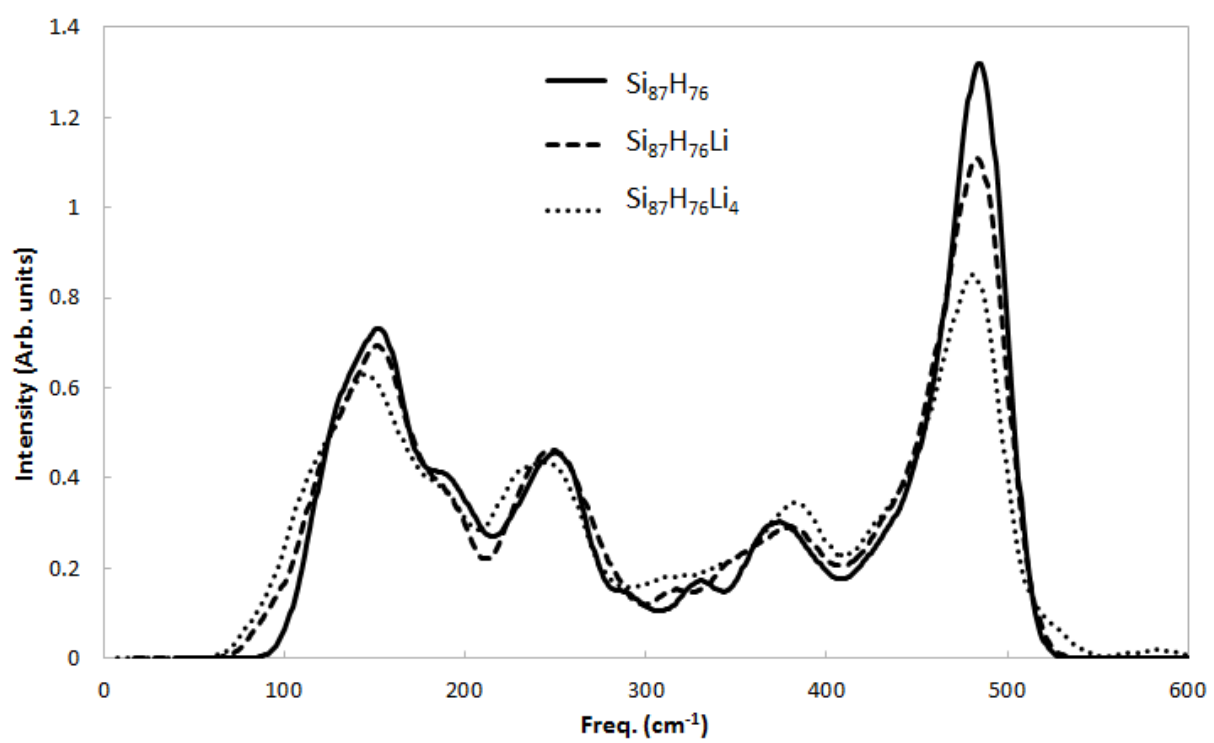

FIG. 4: VDOS for $\mathrm{Si}_{87} \mathrm{H}_{76}$ nanocrystals with zero, one, and four Li dopants, added interstitially near the central Si atom. $\left(10 \mathrm{~cm}^{-1}\right.$ broadening $)$

region of the Si crystal lies partially on the surface of the nanocrystal and experiences a redshift because some of the atoms are undercoordinated. The distinction made between Raman spectra of defects in different locations suggests that Raman spectroscopy can also be used to determine the location of impurities within Si nanocrystals. This is important because previous work by Chan, et al., [23] has shown that nanocrystal size can affect the energetic favorability of Li diffusion into Si nanostructures. 


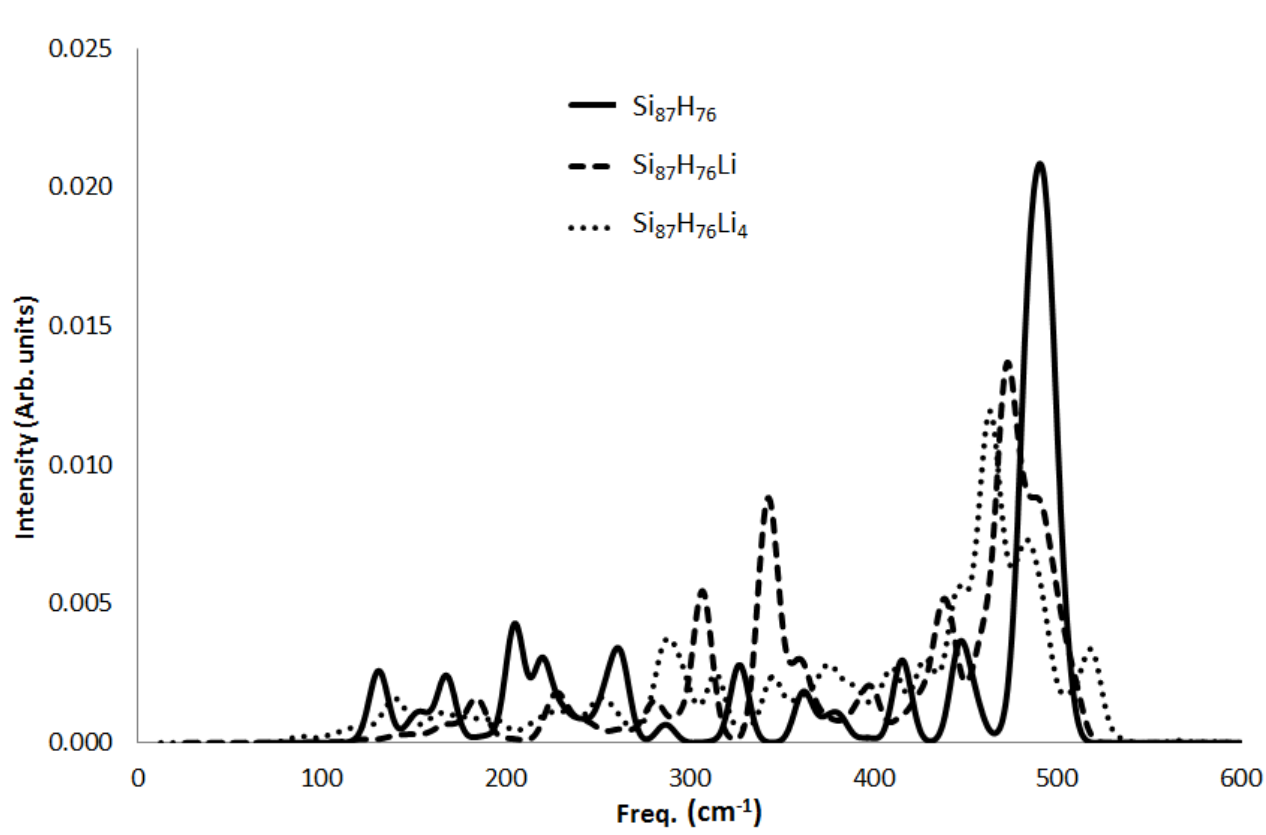

FIG. 5: Calculated Raman shift for $\mathrm{Si}_{87} \mathrm{H}_{76}$ nanocrystals with zero, one, and four Li dopants, added interstitially near the central $\mathrm{Si}$ atom. As the dopant concentration increases, the large peak at $500 \mathrm{~cm}^{-1}$ decreases, indicating disruption of Si crystal lattice. $\left(5 \mathrm{~cm}^{-1}\right.$ broadening $)$

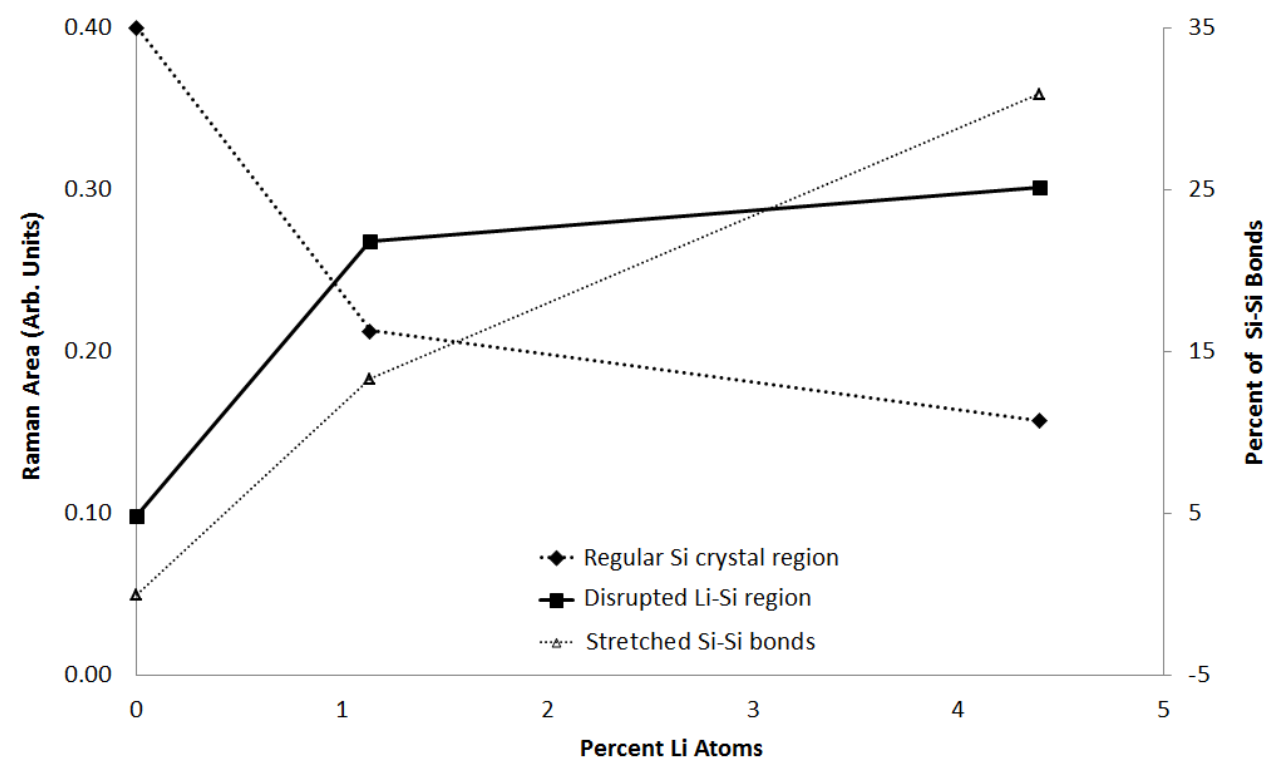

FIG. 6: Area of Raman curve in Si crystal region $\left(480-520 \mathrm{~cm}^{-1}\right)$ and disrupted Si region (440-480 $\mathrm{cm}^{-1}$ ) as a function of Li concentration, given as percent of atoms that are Li. The secondary vertical axis shows the fraction of $\mathrm{Si}-\mathrm{Si}$ bonds that are distorted by more than 0.5 percent from the bondlengths of the intrinsic crystal. 


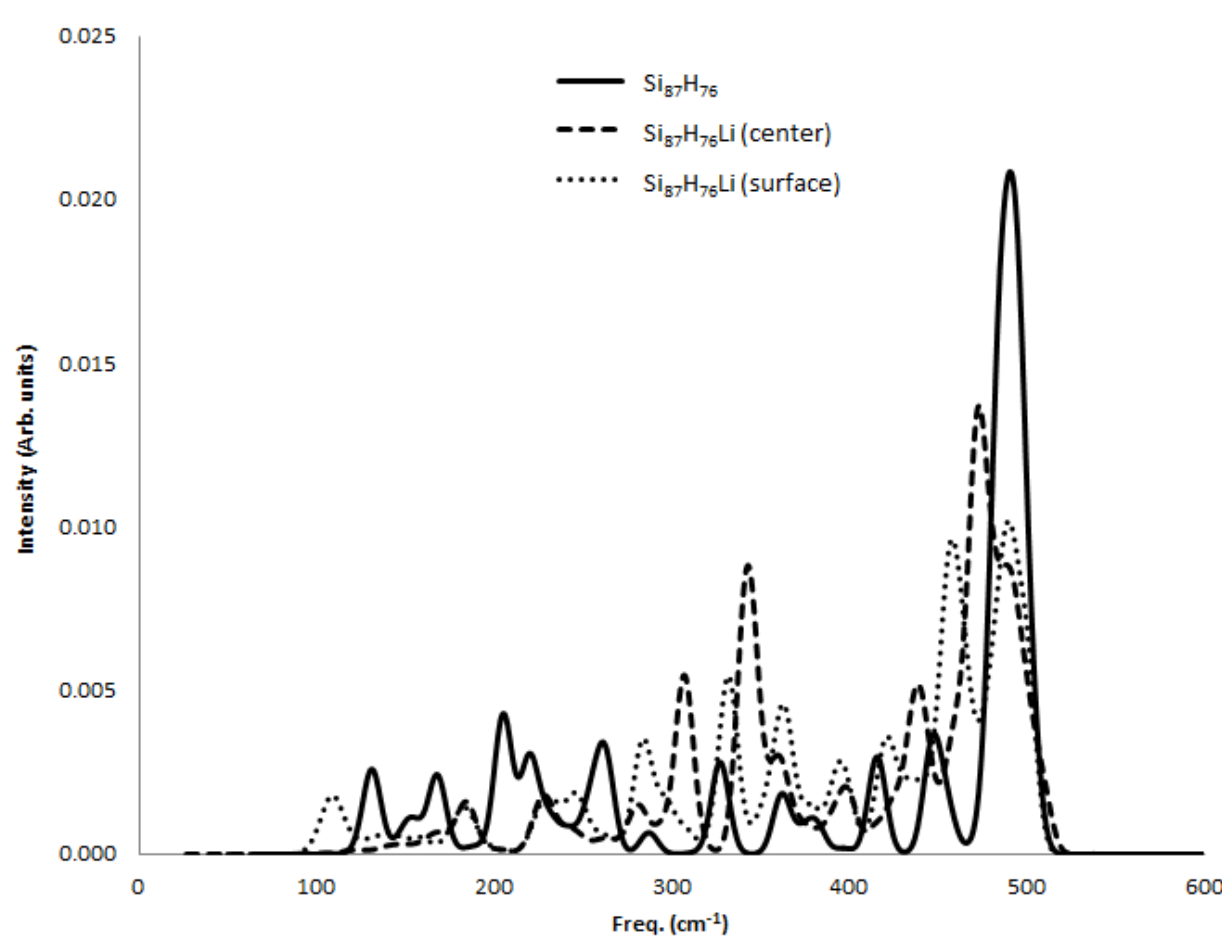

\begin{abstract}
FIG. 7: Calculated Raman shift for $\mathrm{Si}_{87} \mathrm{H}_{76}$ nanocrystals with a single interstitial Li dopant on the surface and near the center of the nanocrystal. The qualitative differences in the Raman spectrum based on the location of the dopant suggest that Raman spectroscopy can be used to characterize the position of impurities in nanocrystals. $\left(5 \mathrm{~cm}^{-1}\right.$ broadening)
\end{abstract}


We examined the effects of Li dopants on the structure of Si nanoscrystals by using first principles techniques to calculate Raman spectra. We calculated the VDOS and Raman spectrum for Si nanocrystals of two different sizes, with various concentration of Li dopants. We also consider variation in the location of the Li dopant, ie., on the surface versus in the center of the nanocrystal. We find that lithiation of the Si nanocrystal lengthens the Si-Si bonds surrounding the interstitial Li dopant and distorts the bond angles associated with these bonds. The result of this distortion is that part of the Si crystal transitions into a locally disrupted region, which is evident by a broad Raman peak that appears from 440-480 $\mathrm{cm}^{-1}$. Furthermore, we find the characteristic Si peak at 500-520 $\mathrm{cm}^{-1}$ exhibits a strong dependence on $\mathrm{Li}$ concentration, where higher $\mathrm{Li}$ concentration reduces the size of this large peak. This suggests that Raman spectroscopy can be used as an indicator of dopant concentration and associated lattice distortion in Si nanocrystals.

\section{ACKNOWLEDGMENTS}

We would like to acknowledge support for our work on nanostructures from grant from the U.S. Department of Energy: DE-FG02-06ER46286. We also wish to acknowledge support provided by the Scientific Discovery through Advanced Computing (SciDAC) program funded by U.S. Department of Energy, Office of Science, Advanced Scientific Computing Research and Basic Energy Sciences under award number DESC0008877 for our work on algorithms. This research used resources of the National Energy Research Scientific Computing Center, a DOE Office of Science User Facility supported by the Office of Science of the U.S. Department of Energy under Contract No. DE-AC02- 05CH11231. The authors gratefully acknowledge helpful discussions with K. H. Khoo.

[1] Szczech, J. R.; Jin, S. Energy Environ. Sci. 2011, 4, 56-72.

[2] Su, X.; Wu, Q.; Li, J.; Xiao, X.; Lott, A.; Lu, W.; Sheldon, B. W.; Wu, J. Advanced Energy Materials 2014, 4, n/a-n/a.

[3] Weydanz, W.; Wohlfahrt-Mehrens, M.; Huggins, R. Journal of power sources 1999, 81, 237242.

[4] Zhang, Q.; Zhang, W.; Wan, W.; Cui, Y.; Wang, E. Nano Letters 2010, 10, 3243-3249. 
[5] Bobbitt, N. S.; Sai, N.; Marom, N.; Kim, M.; Chelikowsky, J. R. The Journal of chemical physics 2014, 141, 094309.

[6] Dalpian, G. M.; Chelikowsky, J. R. Physical review letters 2006, 96, 226802.

[7] Melnikov, D. V.; Chelikowsky, J. R. Physical review letters 2004, 92, 046802.

[8] Li, G.; Ding, K.; Chen, Y.; Han, H.; Wang, Z. Journal of Applied Physics 2000, 88, 1439-1442.

[9] Volodin, V.; Efremov, M.; Gritsenko, V.; Kochubei, S. Applied physics letters 1998, 73, 12121214.

[10] Nesheva, D.; Raptis, C.; Perakis, A.; Bineva, I.; Aneva, Z.; Levi, Z.; Alexandrova, S.; Hofmeister, H. Journal of applied physics 2002, 92, 4678-4683.

[11] Richter, H.; Wang, Z.; Ley, L. Solid State Communications 1981, 39, 625 - 629.

[12] Go, S.; Bilz, H.; Cardona, M. Physical Review Letters 1975, 34, 580.

[13] Hessel, C. M.; Wei, J.; Reid, D.; Fujii, H.; Downer, M. C.; Korgel, B. A. The Journal of Physical Chemistry Letters 2012, 3, 1089-1093.

[14] Umari, P.; Gonze, X.; Pasquarello, A. Phys. Rev. Lett. 2003, 90, 027401.

[15] Zhao, X.; Vanderbilt, D. Phys. Rev. B 2002, 65, 233106.

[16] Rice, C.; Young, R. J.; Zan, R.; Bangert, U.; Wolverson, D.; Georgiou, T.; Jalil, R.; Novoselov, K. S. Phys. Rev. B 2013, 87, 081307.

[17] Bandyopadhyay, A.; Pal, P.; Chowdhury, S.; Jana, D. Materials Research Express 2015, 2, 095603.

[18] Jensen*, L.; ; Schatz, G. C. The Journal of Physical Chemistry A 2006, 110, 5973-5977, PMID: 16671663.

[19] Han, P.; Bester, G. Phys. Rev. B 2012, 85, 041306.

[20] Abdulsattar, M. Silicon 2013, 5, 229-237.

[21] Khoo, K. H.; Zayak, A. T.; Kwak, H.; Chelikowsky, J. R. Phys. Rev. Lett. 2010, 105, 115504.

[22] Khoo, K.; Chelikowsky, J. R. Physical Review B 2014, 89, 195309.

[23] Chan, T.-L.; Chelikowsky, J. R. Nano Letters 2010, 10, 821-825, PMID: 20121259.

[24] Parlinski, K.; Li, Z.; Kawazoe, Y. Physical review letters 1997, 78, 4063.

[25] Porezag, D.; Pederson, M. R. Phys. Rev. B 1996, 54, 7830.

[26] Umari, P.; Pasquarello, A. Diamond and Related Materials 2005, 14, 1255-1261.

[27] Wilson, E. B.; Cross, P. C.; Decius, J. Molecular Vibration: The Theory of Infrared and Raman Vibrational Spectra; Dover New York, 1980. 
[28] Steele, D. Theory of vibrational spectroscopy; Saunders Philidelphia, 1971.

[29] Chelikowsky, J. R.; Troullier, N.; Saad, Y. Physical review letters 1994, 72, 1240.

[30] Chelikowsky, J. R. Journal of Physics D: Applied Physics 2000, 33, R33.

[31] Kronik, L.; Makmal, A.; Tiago, M. L.; Alemany, M.; Jain, M.; Huang, X.; Saad, Y.; Chelikowsky, J. R. phys. stat. solidi (b) 2006, 243, 1063-1079.

[32] Han, J.; Tiago, M. L.; Chan, T.-L.; Chelikowsky, J. R. J. Chem. Phys. 2008, 129, 144109.

[33] Troullier, N.; Martins, J. L. Phys. Rev. B 1991, 43, 1993.

[34] Zhou, Y.; Saad, Y.; Tiago, M. L.; Chelikowsky, J. R. Phys. Rev. E 2006, 74, 066704.

[35] Schofield, G.; Saad, Y.; Chelikowsky, J. R. Quantum algorithms for predicting the properties of complex materials. 2012 .

[36] Ceperley, D. M.; Alder, B. J. Phys. Rev. Lett. 1980, 45, 566-569.

[37] Huang, X.; Lindgren, E.; Chelikowsky, J. R. Physical Review B 2005, 71, 165328.

[38] Boukamp, B.; Lesh, G.; Huggins, R. Journal of the Electrochemical Society 1981, 128, 725729 .

[39] Liu, X. H. et al. Nano Letters 2011, 11, 3312-3318, PMID: 21707052.

[40] Limthongkul, P.; Jang, Y.-I.; Dudney, N. J.; Chiang, Y.-M. Acta Materialia 2003, 51, 11031113.

[41] Li, H.; Huang, X.; Chen, L.; Zhou, G.; Zhang, Z.; Yu, D.; Mo, Y. J.; Pei, N. Solid State Ionics 2000, 135, 181-191.

[42] Holzapfel, M.; Buqa, H.; Hardwick, L. J.; Hahn, M.; Würsig, A.; Scheifele, W.; Novák, P.; Kötz, R.; Veit, C.; Petrat, F.-M. Electrochimica acta 2006, 52, 973-978.

[43] Iqbal, Z.; Veprek, S. Journal of Physics C: Solid State Physics 1982, 15, 377.

[44] Xia, H.; He, Y.; Wang, L.; Zhang, W.; Liu, X.; Zhang, X.; Feng, D.; Jackson, H. E. Journal of applied physics 1995, 78, 6705-6708. 